\title{
Epidemiological analysis of 67 local COVID- 19 clusters in Sichuan Province, China
}

\author{
Suling Mao ${ }^{1 \dagger}$, Ting Huang ${ }^{1 \dagger}$, Heng Yuan ${ }^{1}$, Min Li ${ }^{2}$, Xiaomei Huang ${ }^{3}$, Changxiao Yang $^{1}$, Xingyu Zhou', \\ Xiuwei Cheng ${ }^{1}$, Qian Su${ }^{1}$ and Xianping $\mathrm{Wu}^{1 *}$
}

\begin{abstract}
Background: This study was intended to investigate the epidemiological characteristics of COVID-19 clusters and the severity distribution of clinical symptoms of involved cases in Sichuan Province, so as to provide information support for the development and adjustment of strategies for the prevention and control of local clusters.
\end{abstract}

Methods: The epidemiological characteristics of 67 local clusters of COVID-19 cases in Sichuan Province reported as of March 17, 2020 were described and analyzed. Information about all COVID-19 clusters and involved cases was acquired from the China Information System for Disease Control and Prevention and analyzed with the epidemiological investigation results taken into account.

Results: The clusters were temporally and regionally concentrated. Clusters caused by imported cases from other provinces accounted for $73.13 \%$; familial clusters accounted for $68.66 \%$; the average attack rate was $8.54 \%$, and the average secondary attack rate was 6.11\%; the median incubation period was $8.5 \mathrm{~d}$; a total of 28 cases met the criteria for incubation period determination, and in the 28 cases, the incubation period was $>14 \mathrm{~d}$ in $21.43 \%$ (6/28). a total of 226 confirmed cases were reported in the 67 clusters. Ten cases were exposed before the confirmed cases they contacted with developed clinical symptoms, and the possibility of exposure to other infection sources was ruled out; two clusters were caused by asymptomatic carriers; confirmed cases mainly presented with fever, respiratory and systemic symptoms; a gradual decline in the severity of clinical symptoms was noted with the increase of the case generation.

Conclusions: Population movement and gathering restrictions and strict close contact management measures will significantly contribute to the identification and control of cases. Transmission during the incubation period and asymptomatic infections have been noted. Studies on the pathogenicity and transmissibility in these populations and on COVID-19 antibody levels and protective effects in healthy people and cases are required.

Keywords: Cluster, COVID-19, Epidemiology

\section{Background}

In later December 2019, a number of pneumonia cases of unknown etiology emerged in Wuhan, China. Around the Chinese New Year in 2020, the number of cases rose sharply, involving multiple provinces, municipalities and

\footnotetext{
*Correspondence: rzf080925@163.com

+Suling Mao and Ting Huang contributed equally to this work.

${ }^{1}$ Sichuan Center for Disease Control and Prevention, No. 6 Zhongxue Road, Wuhou District, Chengdu 610,041, Sichuan, China

Full list of author information is available at the end of the article
}

autonomous regions in China; at the same time, outbreaks were reported in many other countries including Japan, Korea, Italy and the United States. On March 11, 2020, the WHO declared COVID-19 a pandemic [1]. On January 16, 2020, the first COVID-19 cluster was reported in Sichuan Province, China; as of March 17, 2020, a total of 67 local clusters were reported, involving 226 confirmed COVID-19 cases. Sichuan Province is a populous province in the southwest of mainland China,

(c) The Author(s). 2020 Open Access This article is licensed under a Creative Commons Attribution 4.0 International License, which permits use, sharing, adaptation, distribution and reproduction in any medium or format, as long as you give appropriate credit to the original author(s) and the source, provide a link to the Creative Commons licence, and indicate if changes were made. The images or other third party material in this article are included in the article's Creative Commons licence, unless indicated otherwise in a credit line to the material. If material is not included in the article's Creative Commons licence and your intended use is not permitted by statutory regulation or exceeds the permitted use, you will need to obtain permission directly from the copyright holder. To view a copy of this licence, visit http://creativecommons.org/licenses/by/4.0/ The Creative Commons Public Domain Dedication waiver (http://creativecommons.org/publicdomain/zero/1.0/) applies to the data made available in this article, unless otherwise stated in a credit line to the data. 
so absence of stringent and effective prevention and control could have resulted in serious social consequences. We analyzed the epidemiological characteristics of COVID-19 clusters in Sichuan, intended to provide information support for the development and adjustment of local prevention and control strategies.

\section{Methods}

\section{Study design}

The epidemiological characteristics of 67 local COVID-19 clusters in Sichuan reported as of March 17, 2020 and confirmed cases involved in these clusters were described and analyzed using a cross-sectional study design.

\section{Data source}

In accordance with requirements in the Guidance for Corona Virus Disease 2019: Prevention, Control, Diagnosis and Management [2], information about all COVID19 clusters and cases should be reported to the China Information System for Disease Control and Prevention. In this study, information about all local clusters was exported from sub-module "Emergency Public Reporting System" and data about related confirmed cases and asymptomatic carriers from sub-module "Infectious Disease Management Information System". Based on epidemiological investigation results, transmission chains were constructed and case generations were determined, followed by a comprehensive analysis. This study has covered all local clusters and all confirmed cases and asymptomatic carriers in the clusters, so sampling was not involved. Confirmed cases with missing clinical information were not included into the analysis.

\section{Definitions}

Confirmed cases, asymptomatic carriers and clusters were identified according to the definitions in the Guidance for Corona Virus Disease 2019: Prevention, Control, Diagnosis and Management [2]. A cluster outbreak indicated that more than two confirmed cases or asymptomatic carriers were found within 14 days in a small area (such as a family, a building site, a work unit), and there was a possibility of human-to-human transmission caused by close contact or by exposure to infectious source together. All confirmed cases were divided into mild cases (the clinical symptoms are mild and no pneumonia manifestation can be found in imaging); ordinary cases (with symptoms like fever and respiratory tract symptoms, and pneumonia manifestation can be seen in imaging); and severe cases (respiratory distress, $R R \geq 30$ breaths/min; pulse oxygen saturation $(\mathrm{SpO} 2) \leq 93 \%$ on room air at rest state; arterial partial pressure of oxygen $(\mathrm{PaO} 2)$ /oxygen concentration $(\mathrm{FiO} 2) \leq 300 \mathrm{mmHg}$. The date of onset for a confirmed case was defined as the date of first appearance of clinical symptoms self- reported in field epidemiological investigation. The date of onset for an asymptomatic carrier was defined as the date on which a positive COVID-19 pathological test was obtained with respiratory tract or other feasible samples.

The attack rate was defined as the number of cases divided by the number of exposed persons, where the number of cases was the total number of confirmed cases involved in a transmission chain. As both secondgeneration (G2) and third-generation (G3) cases were identified from close contacts of cases of the previous generations, if any G2 case developed in a cluster, the number of exposed persons was the number of close contacts of first-generation (G1) cases plus the number of G1 cases; if any G3 case developed in a cluster, the number of exposed persons was the number of close contacts of G1 cases plus the number of close contacts of $\mathrm{G} 2$ cases resulting in the $\mathrm{G} 3$ cases plus the number of G1 cases; and so forth. The secondary attack rate was defined as the number of second- and later-generation cases divided by the number of exposed persons, where the number of exposed persons was calculated using the aforementioned algorithm except that the number of G1 cases was not included. The incubation period was determined based on confirmed cases that had been exposed for a single time in transmission chains, whose exposure and onset time were clearly known and in whom other factors potentially responsible for their infection were ruled out. Cases for the determination of the attack rate, the secondary attack rate and the incubation period included confirmed cases and asymptomatic carriers. A family dinner was defined as a dinner attended by two or more families.

\section{Statistical analyses}

Epidemiological characteristics of clusters and demographic and clinical characteristics of confirmed cases were descriptively analyzed. Attack rates, secondary attack rates and clinical types of cases and constituent ratios of symptoms were compared using $x^{2}$ test for constituent differences. The regional distribution map in Fig. 2 was created by geocoding all COVID-19 clusters and matching them to the city-level layers of polygon and point by administrative codes with the use of ArcGIS software.

\section{Results}

\section{Distribution of clusters}

On January 16, 2020, the first local COVID-19 cluster was reported in Sichuan. On January 24, 2020, a level-1 public health emergency response was launched, followed by the successive execution of multiple measures with respect to infection sources, routes of transmission and susceptible populations to effectively reduce 
population movement and gatherings and strengthen the management of close contacts. Main measures and the timeline are shown in Fig. 1.

On January 16, 2020, the first local COVID-19 cluster was reported. A total of 31 clusters were reported in January and 36 in February. The daily number of clusters peaked on January 30, $2020(n=7)$ and gradually declined after February 06, 2020. A total of 50 (74.63\%) clusters were reported as of February 06, 2020. Temporal distribution of cluster reports is shown in Fig. 1. Among the clusters, 49 (73.13\%) were caused by imported cases, and in 18 the infection source of the first case was unknown.

The 67 clusters had involved 16 prefectures and 48 districts/counties (Fig. 2). Chengdu had the largest number of clusters $(n=17)$, followed by Dazhou $(n=12)$, Nanchong $(n=7)$ and Guang'an $(n=6)$. Clusters in the four prefectures accounted for $62.69 \%(42 / 67)$ of the total in the province.

Households were the primary exposure place in the reported clusters, accounting for $68.66 \%$ (46/67); living in the same household was the primary form of exposure, accounting for $60.87 \%$ (28/46). Clusters caused by exposure in multiple places and in multiple forms accounted for $11.94 \%$ (Fig. 3). Significantly more clusters were caused by family gatherings in February than in January $(p=0.002)$ (Table 1$)$.

\section{Characteristics of confirmed cases involved in clusters}

In the 67 clusters, a total of 226 confirmed cases were reported, accounting for $42.01 \%$ (226/538) of the total in the whole province; among these confirmed cases, 164 were local confirmed cases resulting from clusters, accounting for $72.57 \%$ (164/226); the male/female ratio was $1: 1$, the mean age was 47.56 years (1 month- 87 years), and patients 30 years of age or above accounted for $87.17 \%$ (197/226); involved were 75 (33.19\%) G1 cases, 131 (57.96\%) G2 cases and 20 (8.85\%) G3 cases (Table 2); $23.01 \%(52 / 226)$ had a history of travel or residence in Wuhan, and $25.66 \%(58 / 226)$ had a history of travel or residence in other provinces except Wuhan. The constituent ratios of cases with a history of travel or residence in both Wuhan $(p<0.001)$ and other provinces except Wuhan $(p=0.006)$ were higher in G1 cases were higher in G2 and G3 cases, and the differences were statistically significant (Table 3 ).

The 226 confirmed cases were clinically typed as follows: $65.04 \%(147 / 226)$ were ordinary, $26.55 \%(60 / 226)$ were mild, and $8.41 \%$ (19/226) were severe; no death occurred. With the increase of the transmission generation,

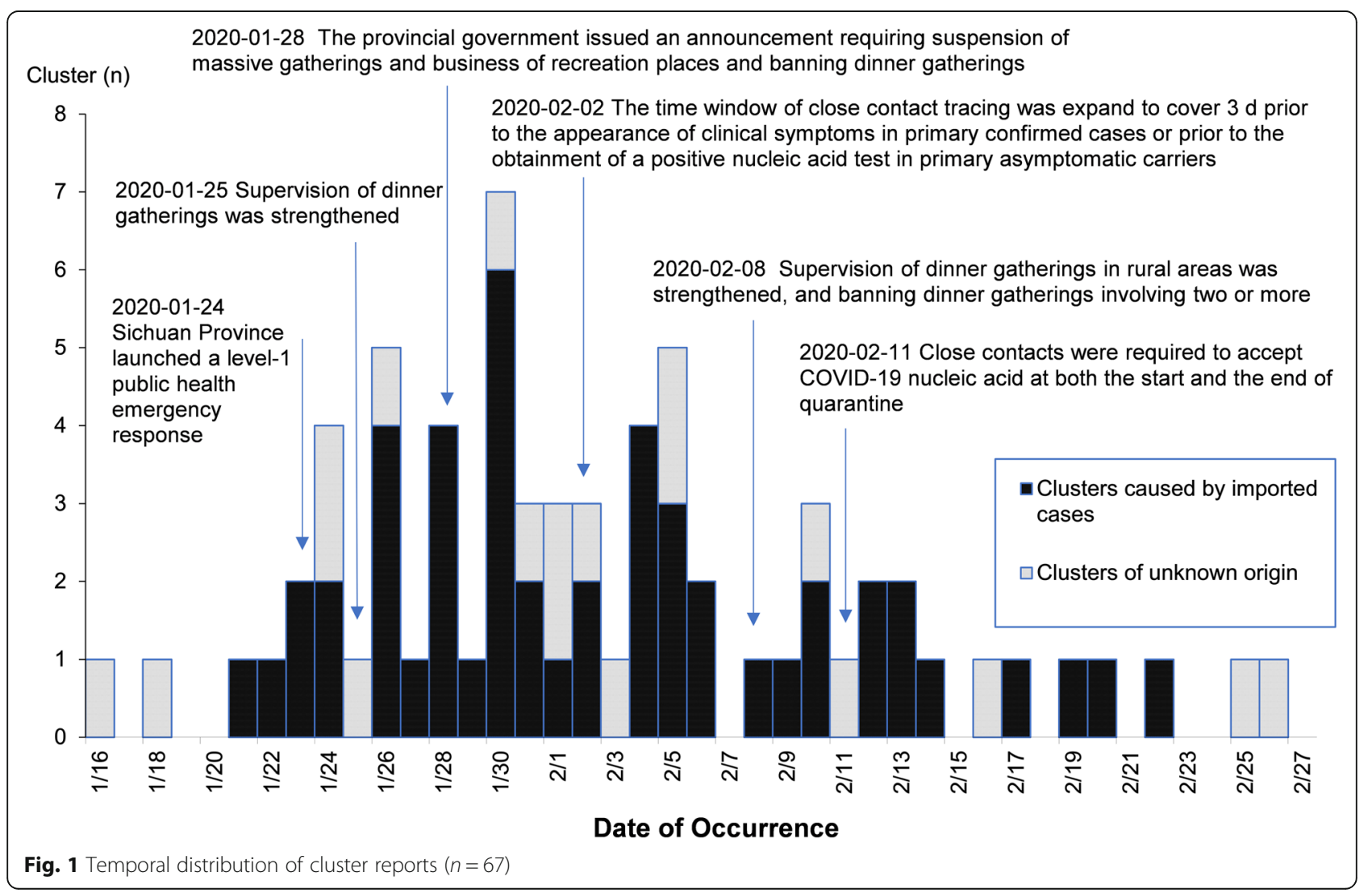




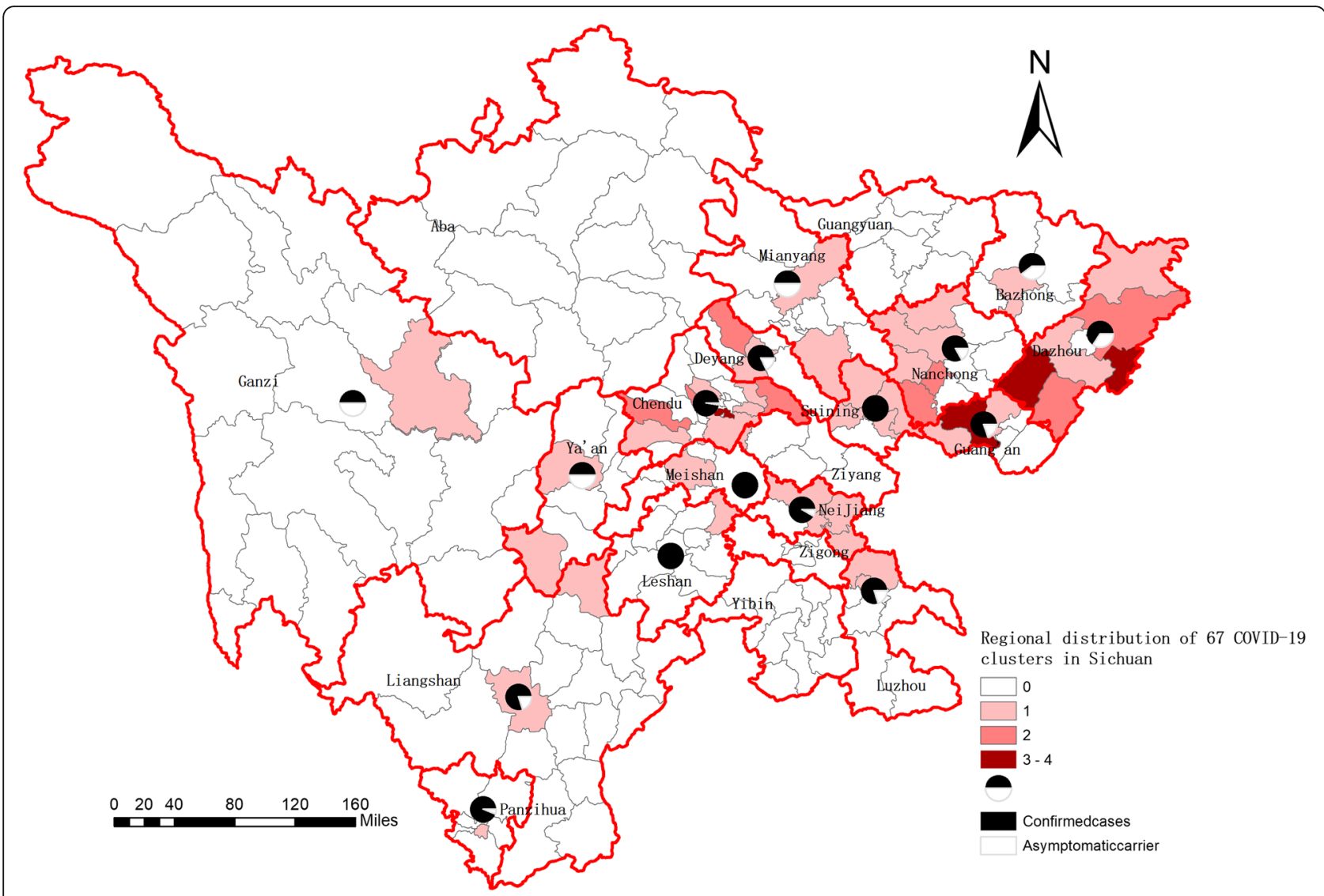

Fig. 2 Regional distribution of 67 COVID-19 clusters in Sichuan. This figure was originally generated by using open access map layers available from National Geomatics Center of China (http://www.ngcc.cn/ngcc/html/1/index.html)

the number of ordinary cases gradually decreased, with a significant difference noted overall $(p=0.045)$; a significant difference was found between G1 and G3 cases in the constituent ratio of ordinary cases $(p=0.013)$. No apparent between-generation difference was found in the constituent ratios of mild or severe cases (Fig. 4).

Among the 226 cases, clinical information about 221 was complete. One hundred thirty-seven cases had fever, accounting for $61.99 \%$, with a median maximum temperature of $38.09^{\circ} \mathrm{C}\left(37.3-39.5^{\circ} \mathrm{C}\right)$. Other main symptoms included dry cough, [66.52\% (147/221)], sputum production $[26.24 \%(58 / 221)]$, fatigue [25.34\% (56/ $221)]$, sore throat $[19.46 \%(43 / 221)]$ and headache [17.19\% (38/221)]. Gastrointestinal symptoms were occasional, mainly including nausea $[10.96 \%(24 / 221)]$ and diarrhea $[6.79 \%(15 / 221)]$.The number of cases with fever showed a declining trend overall with the increase of transmission generation $(p=0.019)$, and significant differences were noted between G1 and G2 cases ( $p=$ $0.009)$ as well as G3 cases $(p=0.027)$ in terms of the constituent ratio of cases with fever. No statistically significant difference was found between generations in other symptoms (Fig. 5).

\section{Transmission characteristics}

Among the 67 clusters, G2 cases were identified in 57 (85.07\%) and G3 cases in 10 (14.93\%); a total of 267 confirmed cases (including 41 asymptomatic carriers) were reported, and on average each cluster resulted in 3.99 $(2-17)$ cases; the average attack rate was $8.54 \%(1.02-$ $100 \%)$, and the average secondary attack rate was $6.11 \%$ (0.51-66.67\%).

Starting from February 02, 2020, we expanded the time window of close contact tracing to cover 3 days prior to the appearance of clinical symptoms in primary confirmed cases or prior to the obtainment of a positive nucleic acid test in primary asymptomatic carriers. Attack rates and secondary attack rates before and after this time point were compared, and it was found that, following the expansion of the time window of close contact tracing, both the attack rate and the secondary attack rate increased, with statistical differences identified (Table 4).

\section{Timing of key events}

Among the 226 cases, 78.76\% (178/226) had been exposed to confirmed cases. In $82.02 \%(146 / 178)$ of such 


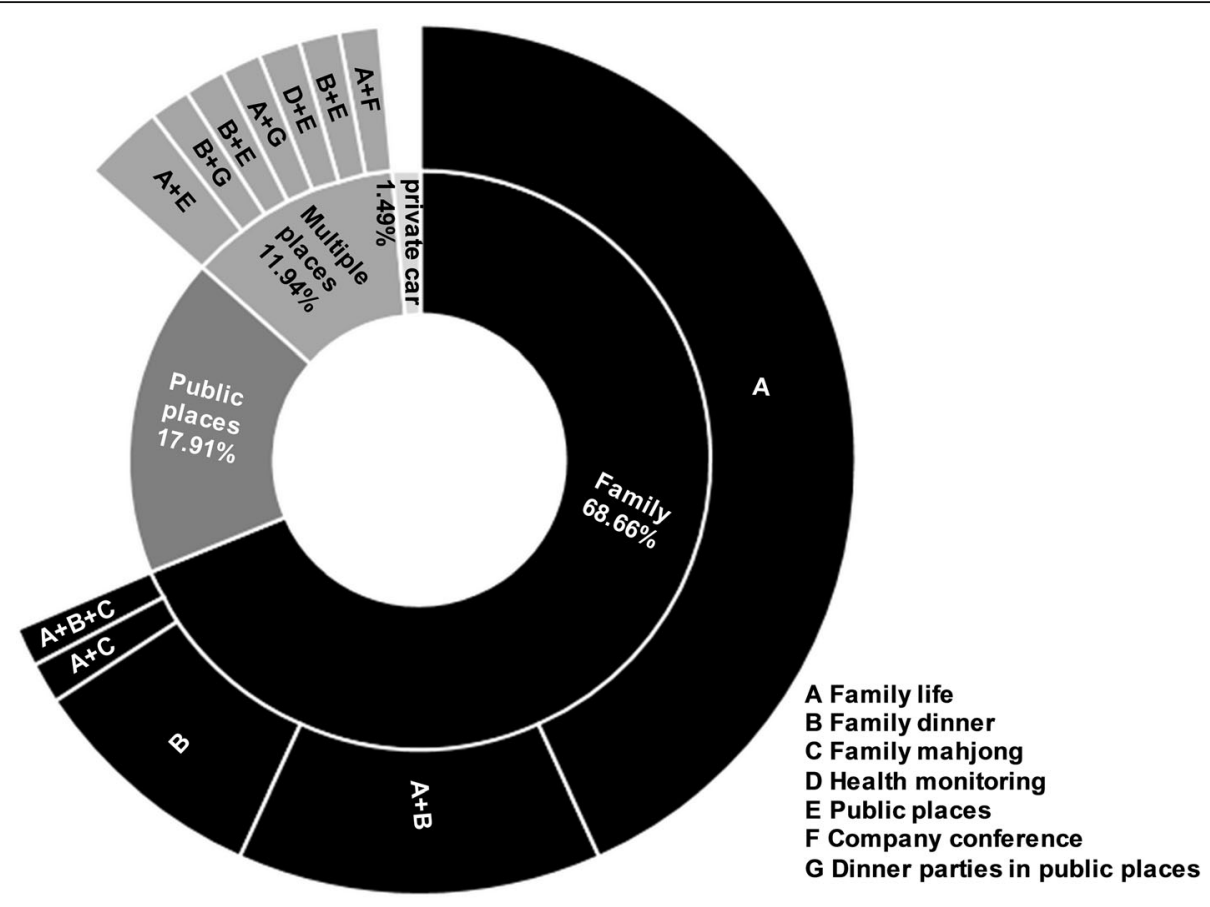

Fig. 3 Distribution of exposure places and forms in clusters

cases, the exposure to the confirmed cases occurred at or after onset of the disease in them. In 10 cases (involved in 9 clusters), the exposure occurred before the confirmed cases they contacted with developed clinical symptoms, and they had been exposed only once while the possibility of exposure to other infection sources could be ruled out. The median time of exposure was 3 d (1-4 d) prior to disease onset in the confirmed cases they contacted with, and the mean was $2.5 \mathrm{~d} ; 90.00 \%$ (9/ 10) of the cases were exposed within $3 \mathrm{~d}$ prior to disease onset in the confirmed cases they contacted with.

Of 226 confirmed cases, the median interval between hospital visit and onset was $1 \mathrm{~d}(0-23 \mathrm{~d})$, the median interval between confirmation and onset was $5 \mathrm{~d}(0-27$ d), and the median interval between isolation and confirmation was $-2 \mathrm{~d}(-19-0 \mathrm{~d})$. Twenty-eight cases met the criteria for incubation period determination (involved in 17 clusters). The average incubation period was $10.3 \mathrm{~d}$, the median incubation period was $8.5 \mathrm{~d}$ (1-
$24 \mathrm{~d})$ and the incubation period was $>14 \mathrm{~d}$ in $21.43 \%(6 /$ 28).

\section{Asymptomatic carriers}

In the 67 clusters, a total of 41 asymptomatic carriers (all were identified during medical observation of close contacts) were reported, including 23 males and $18 \mathrm{fe-}$ males. The mean age was 35.31 years ( 8 months- 81 years); and patients 30 years of age or above accounted for $70.73 \%$ (29/41). Involved were 8 (19.51\%) G1 cases, 31 (75.61\%) G2 cases and 2 (4.88\%) G3 cases.

In one cluster, one G1 case (severe) was found to have spread the disease to one G2 case (mild) and one G3 case (asymptomatic). Two clusters were caused by asymptomatic carriers and, in both clusters, only G2 cases developed, including one (asymptomatic) to two (confirmed) transmission in one cluster and one (asymptomatic) to one (confirmed) transmission in another cluster. The possibility of other infections was ruled out

Table 1 Distribution of clusters caused by family gatherings in January and February

\begin{tabular}{lllllll}
\hline No. (\%) & Total & $\begin{array}{l}\text { Family } \\
\text { dinner }\end{array}$ & $\begin{array}{l}\text { Family } \\
\text { life }\end{array}$ & $\begin{array}{l}\text { Family life + family } \\
\text { mahjong }\end{array}$ & $\begin{array}{l}\text { Family life + family } \\
\text { dinner }\end{array}$ & $\begin{array}{l}\text { Family life + family dinner + family } \\
\text { mahjong }\end{array}$ \\
\hline January & $\begin{array}{l}15 \\
(32.61)\end{array}$ & $1(6.67)$ & $11(73.33)$ & $1(6.67)$ & $2(13.33)$ & $0(0.00)$ \\
February & $\begin{array}{l}31 \\
(67.39)\end{array}$ & $6(19.35)$ & $17(54.84)$ & $0(0.00)$ & $7(22.58)$ & $1(3.23)$ \\
\hline
\end{tabular}


Table 2 Distribution of confirmed cases in 67 clusters, by age and gender

\begin{tabular}{|c|c|c|c|c|c|c|c|c|}
\hline & \multirow{2}{*}{$\begin{array}{l}\text { Total } \\
\text { no. }\end{array}$} & \multirow{2}{*}{$\begin{array}{l}\text { Constituent } \\
\text { ratio (\%) }\end{array}$} & \multicolumn{6}{|c|}{ Case generation } \\
\hline & & & G1 (no.) & Constituent ratio (\%) & G2 (no.) & Constituent ratio (\%) & G3 (no.) & Constituent ratio (\%) \\
\hline \multicolumn{9}{|c|}{ Age group (yrs) } \\
\hline$<20$ & 8 & 3.54 & - & - & 8 & 100 & - & - \\
\hline $20-$ & 21 & 9.29 & 2 & 9.52 & 17 & 80.95 & 2 & 9.52 \\
\hline $30-$ & 43 & 19.03 & 19 & 44.19 & 21 & 48.84 & 3 & 6.98 \\
\hline $40-$ & 59 & 26.11 & 24 & 40.68 & 32 & 54.24 & 3 & 5.08 \\
\hline $50-$ & 47 & 20.80 & 16 & 34.04 & 24 & 51.06 & 7 & 14.89 \\
\hline$\geq 60$ & 48 & 21.24 & 14 & 29.17 & 29 & 60.42 & 5 & 10.42 \\
\hline \multicolumn{9}{|l|}{ Gender } \\
\hline Male & 113 & 50.00 & 43 & 38.05 & 61 & 53.98 & 9 & 7.96 \\
\hline Female & 113 & 50.00 & 32 & 28.32 & 70 & 61.95 & 11 & 9.73 \\
\hline
\end{tabular}

in all G2 cases. By the end of the two clusters, the G1 asymptomatic carriers had not presented with clinical symptoms or imaging abnormalities.

\section{Discussion}

Our analysis of COVID-19 clusters in Sichuan reveals that the majority of clusters were reported before February 06 and occurred in four regions of Sichuan, and three-fourth of the clusters were caused by importations from other provinces including Wuhan. The study also found that the proportion of cases with a history of travel or residence in Wuhan was significantly higher in G1 cases than in secondary cases and the clusters mainly occurred in households with a greater proportion in February than in January. These characteristics were similar to clusters in other provinces in China [3].

The above characteristics of COVID-19 clusters were supposed to be related with several factors. Firstly, the epidemic happened during the Spring Festival in China, when large numbers of people in China returned home for family reunion or visited their friends or relatives, forming a population shift peak, particularly in the midlate January. Secondly, Chengdu, the capital of Sichuan Province, as a rapidly growing metropolis in West China, has well-developed transport infrastructure including highways, railways and airlines, which facilitated the spread of the disease [4]; big data analysis also showed that Chengdu was ranked the top in terms of inflow of people from Wuhan [5-7]; Dazhou and Guang'an, located in the northeast of Sichuan, are adjacent to Chongqing, which was relatively hardly hit by the epidemic among provinces other than Hubei in China [8]. Finally, on January 24, 2020, Sichuan launched a level-1 public health emergency response $[9,10]$, followed by the successive execution of multiple measures with respect to infection sources, routes of transmission and susceptible populations, effectively reducing population movement and gatherings and strengthening the management of close contacts. Specifically, on January 25, 2020, the Administration for Market Regulation, the Health Commission and the Department of Commerce of Sichuan Province jointly issued a document [11] for strengthening the supervision of dinner gatherings; on January 28, 2020, the People's Government of Sichuan Province issued an order [12] requiring suspension of massive gatherings and business of recreational places and banning dinner gatherings. On February 8, 2020, the provincial headquarters for emergency response issued a notice on strengthening the supervision of dinner gatherings in rural areas [13], banning dinner gatherings involving two or more families. The above measures have effectively reduced people's gatherings including festival

Table 3 Travel or residence history of 226 confirmed cases

\begin{tabular}{|c|c|c|c|c|c|}
\hline \multirow{2}{*}{$\begin{array}{l}\text { Case } \\
\text { Generation }\end{array}$} & \multirow{2}{*}{$\begin{array}{l}\text { Cases } \\
\text { (n) }\end{array}$} & \multicolumn{2}{|l|}{ Wuhan } & \multicolumn{2}{|c|}{ Other Provinces except Wuhan } \\
\hline & & Cases (no.) & Constituent Ratio (\%) & Cases (no.) & Constituent Ratio (\%) \\
\hline G1 & 75 & 45 & 60.00 & 29 & 38.67 \\
\hline G2 & 131 & 7 & 5.34 & 26 & 19.85 \\
\hline \multirow[t]{2}{*}{ G3 } & 20 & - & - & 3 & 15.00 \\
\hline & & $x^{2}=75.50$ & $p<0.001$ & $x^{2}=10.16$ & $P=0.006$ \\
\hline Total & 226 & 52 & 23.01 & 58 & 25.66 \\
\hline
\end{tabular}


Table 4 Comparison of attack rates and secondary attack rates in clusters before and after February 02, 2020

\begin{tabular}{|c|c|c|c|c|c|c|c|c|}
\hline Time & $\begin{array}{l}\text { No. of } \\
\text { cases }\end{array}$ & $\begin{array}{l}\text { No. of exposed } \\
\text { persons }\end{array}$ & $\begin{array}{l}\text { Attack rate } \\
(\%)\end{array}$ & $\begin{array}{l}p \\
\text { value }\end{array}$ & $\begin{array}{l}\text { No. of secondary } \\
\text { cases }\end{array}$ & $\begin{array}{l}\text { No. of exposed } \\
\text { persons }\end{array}$ & $\begin{array}{l}\text { Secondary attack } \\
\text { rate }(\%)\end{array}$ & $\begin{array}{l}p \\
\text { value }\end{array}$ \\
\hline $\begin{array}{l}\text { Before February 02, } \\
2020\end{array}$ & 156 & 2027 & 7.70 & 0.022 & 107 & 1990 & 5.38 & 0.022 \\
\hline $\begin{array}{l}\text { After February 02, } \\
2020\end{array}$ & 111 & 1100 & 10.09 & & 80 & 1072 & 7.46 & \\
\hline
\end{tabular}

${ }^{\mathrm{a}}$ Both the number of cases and the number of secondary cases include confirmed cases and asymptomatic carriers

celebrations and company dinner parties. However, still a minority of people, following traditional customs, organized and participated in family dinners and entertainment activities with inadequate protection during the Chinese New Year, resulting in the familial clustering characteristic of the epidemic.

The attack rate of COVID-19 clusters in our study was slightly lower than $9.6 \%$ in a study located in Shenzhen [14]. The interval between hospital visit and onset was shorter than that reported in Hunan [15]; related data were not available from other provinces. The study also showed that the attack rate and the secondary attack rate were significantly higher after February 02, 2020 than before; cases were mainly confirmed during the isolation period and asymptomatic carriers were identified in the management of close contacts, which suggested that the intensive close contact management measures in Sichuan including expansion of the close contact tracing time window to cover $3 \mathrm{~d}$ prior to the presence of clinical symptoms (or positive test for asymptomatic carriers) in the confirmed cases they contacted with, implemented from February 02, 2020, and the conduct of nucleic acid testing at both the start and the end of quarantine of close contacts, implemented from February 11, 2020, might have contributed to the discovery and control of cases and the reduction of spread.

The median incubation period in the limited cases in Sichuan was corresponding to the finding in other studies in China that the incubation period in the majority of cases was $<14 \mathrm{~d}$, but being longer than the median incubation period reported in other studies (3-7 d) [16-20]. It was also found that the proportion of cases with an incubation period $>14 \mathrm{~d}$ was higher than that reported in a study in Henan (7.45\%) [21]. Ten cases exposed before the confirmed cases they contacted with presented with clinical symptoms, of which most cases exposed within 3 $\mathrm{d}$ prior to disease onset, and these cases had been exposed only once and other potential sources of infection could be ruled out, suggesting COVID-19 is possibly transmittable during the incubation period, similar to a report in Zhejiang [22]. At the same time, the majority of cases involved in the clusters exposed within 3 days prior to onset of the disease in confirmed cases they contacted with, suggesting COVID-19, like influenza, is possibly contagious at the end of the incubation period or tracing back to $3 \mathrm{~d}$ prior to disease onset in seeking close contact could miss earlier infections in the incubation period. What's more, two clusters caused by

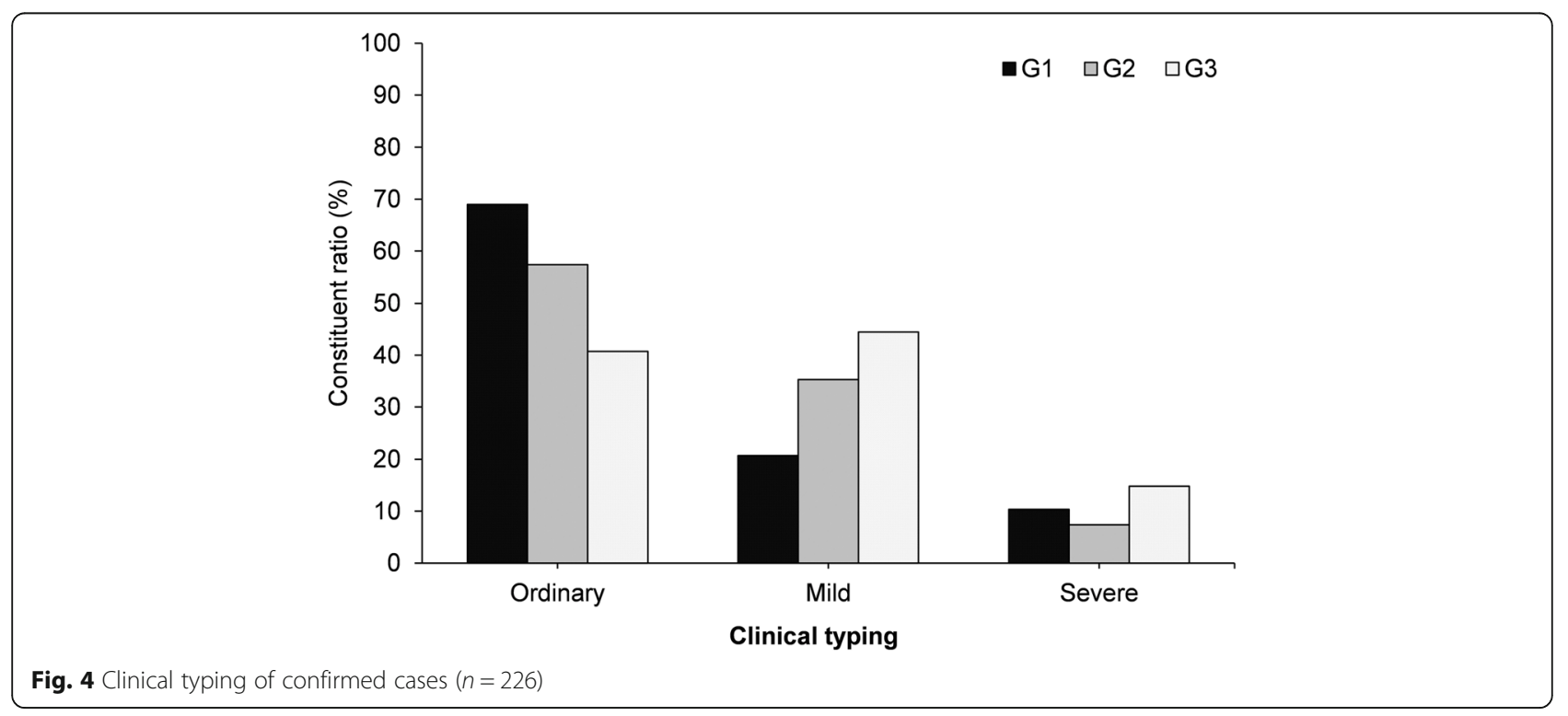




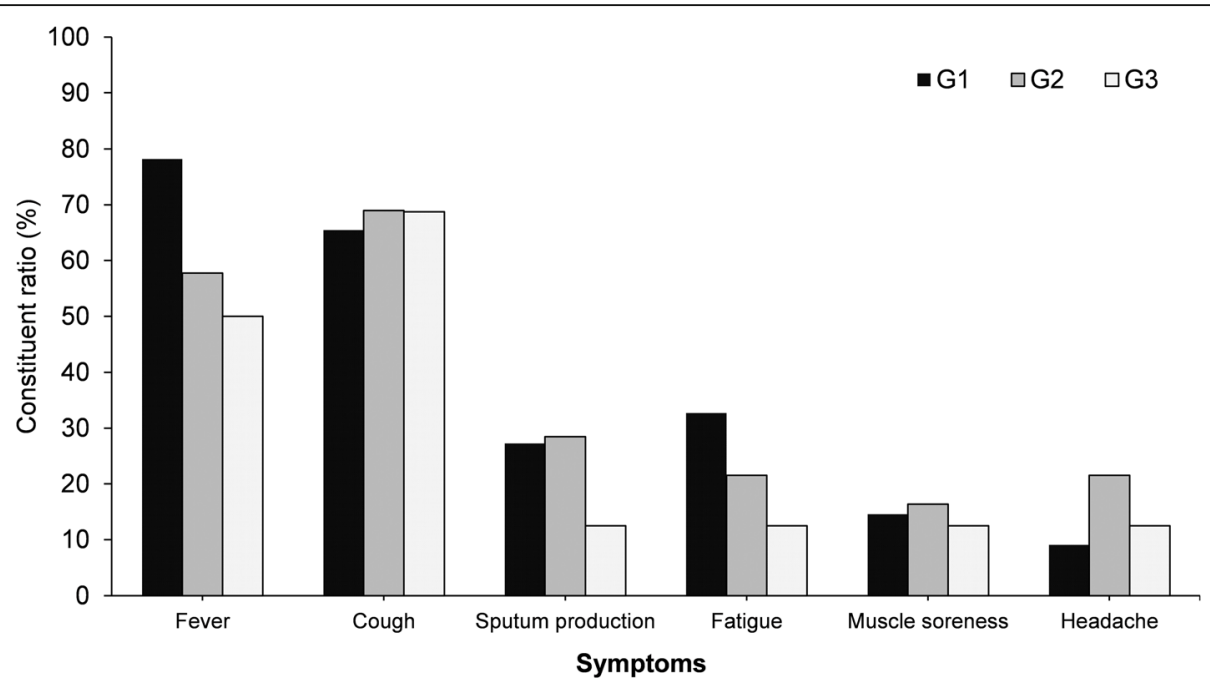

Fig. 5 Main clinical symptoms of confirmed cases $(n=221)$

asymptomatic carriers indicated COVID-19 could be transmitted by asymptomatic infected persons, as is similar to the report in Henan [21]. Nevertheless, the asymptomatic carriers had only caused a small number of G2 cases, indicating the transmissibility in this population may be limited. The above two populations cannot be effectively identified and thus are of special public health significance for sustained transmission of COVID-19 [23]; due to limited available data, the transmissibility of the disease cannot be analyzed, and studies on its pathogenicity and transmissibility in these populations are awaited.

Main presentations of confirmed cases involved in the clusters in Sichuan included fever, respiratory and systemic symptoms, basically corresponding to the reported clinical characteristics of COVID-19 cases, and no characteristic clinical symptom was seen [18, 24-27]. In clusters, the proportion of ordinary cases and cases with fever decreased with the increase of the case generation, suggesting that the severity of clinical symptoms might gradually lighten with the development of the epidemic. This corresponded to reports that, in the initial period of the epidemic, cases were mainly severe and the case fatality rate was high [18] and, in the later period of the epidemic, cases were mainly mild [26]. This is possibly because the virus will maintain moderate virulence during passage and evolution for the purpose of long-time reproduction in human and the infection was limited to the upper respiratory tract [28]. In addition, it was reported that antibody levels would not last long, and repeated infection was common [29,30]. However, limited data are available and further studies on COVID-19 antibody levels and protective effects are awaited for investigating whether post-infection antibody levels will decline following the mitigation of COVID-19 clinical symptoms or whether repeated infection will occur as a result of the short-term maintenance of antibody levels.

The findings in the study are subject to several limitations. On the one hand, as only cases exposed only once were included, the cases calculated for the incubation period was limited. On the other hand, PCR detection of respiratory tract specimens was carried out once each time when close contacts were isolated and released, and the isolation period was 14 days. If an asymptomatic carrier recovered spontaneously during the isolation period, the attack rate might be underrated due to the missing count of cases.

\section{Conclusions}

In conclusion, our study demonstrated the comprehensive epidemic situation of COVID-19 in Sichuan Province and confirmed the infectivity during the incubation period and asymptomatic infection, providing a reference for decision makers to formulate and adjust control measures.

\section{Abbreviations}

WHO: World health organization; COVID-19: Coronavirus disease; RR: Respiratory rate; $\mathrm{SpO} 2$ : Pulse oxygen saturation; $\mathrm{PaO} 2$ : Arterial partial pressure of oxygen; FiO2: Fraction of inspiration $\mathrm{O} 2$; G1: First generation; G2: Second generation; G3: Third generation; PCR: Polymerase chain reaction

\section{Acknowledgments}

We salute and thank all personnel engaged in the prevention, control, diagnosis and treatment of COVID-19 in Sichuan Province.

\section{Authors' contributions}

Study concept and design, analysis and interpretation of data, preparation of manuscript, and supervision: SM, TH, XW. Acquisition of data: ML, XH, XC. Analysis and interpretation of data: $\mathrm{HY}, \mathrm{XZ}$. Preparation of manuscript: $C Y, \mathrm{QS}$. All authors have read and approved the manuscript. 


\section{Funding}

This study was supported by the Science and Technology Department of Sichuan Province (grant no. 2020YFS0015). The funder of the study had no role in study design, data collection, data analysis, data interpretation, or writing of the report.

\section{Availability of data and materials}

The datasets used and/or analysed during the current study are available from the corresponding author on reasonable request.

\section{Ethics approval and consent to participate}

This study was consulted to the ethics committees of Sichuan Center for Disease Control and Prevention. Ethics approval was not available because all data were collected as part of public health emergency surveillance following the Laws of the People's Republic of China on the Prevention and Treatment of Infectious Diseases. We also did not include any data of patients' personal information and therefore ethical approval was not required and waived off written informed consent.

\section{Consent for publication}

Not applicable.

\section{Competing interests}

The authors declare that they have no competing interests.

\section{Author details}

${ }^{1}$ Sichuan Center for Disease Control and Prevention, No. 6 Zhongxue Road, Wuhou District, Chengdu 610,041, Sichuan, China. ${ }^{2}$ Jianyang Center for Disease Control and Prevention, Chengdu, Sichuan, China. ${ }^{3}$ Panzhihua Center for Disease Control and Prevention, Panzhihua, Sichuan, China.

Received: 2 May 2020 Accepted: 24 September 2020

Published online: 08 October 2020

\section{References}

1. WHO. WHO Director-General's opening remarks at the media briefing on COVID-19. 2020. https://www.who.int/dg/speeches/detail/who-directorgeneral-s-opening-remarks-at-the-media-briefing-on-covid-19---11-march2020. Accessed 17 Mar 2020.

2. National Health Commission (NHC) of the PRC, National Administration of Traditional Chinese Medicine of the PRC. Guidance for Corona Virus Disease 2019: Prevention, control, Diagnosis and Management People's Medical Publishing House; 2020.

3. Special Expert Group for Control of the Epidemic of Novel Coronavirus Pneumonia of the Chinese Preventive Medicine Association. An update on the epidemiological characteristics of novel coronavirus pneumonia (COVID19) [in Chinese]. Chin J Epidemiol. 2020;41(2):139-144. https://doi.org/ https://doi.org/10.3760/cma.j.issn.0254-6450.2020.02.002.

4. Yang HY, Duan GC. Analysis on the epidemic factors for the Corona virus disease [in Chinese]. Chin J Prev Med 2020;54. https://doi.org/https://doi. org/10.3760/cma.j.cn112150-20200227-00196.

5. How Internet of things big data aid in epidemic surveillance: Where did Wuhan people go the day before the city "lockdown"? https://www. gongkongke.com/posts/caNo4iyJUzNz/. Accessed 17 Mar 2020.

6. Baidu Echarts. http://qianxi.baidu.com/. Accessed 17 Mar 2020.

7. Hu JX, He GH, Liu T, Xiao JP, Rong ZH, Guo LC, et al. Risk assessment of exported risk of novel coronavirus pneumonia from Hubei Province [in Chinese]. Chin J Prev Med2020;54(4):1-5. https://doi.org/https://doi.org/10. 3760/cma.j.cn112150-20200219-00142.

8. Chinese Center for Disease Control and Prevention. Distribution of COVID19 Cases. http://2019ncov.chinacdc.cn/2019-nCoV/. Accessed 17 Mar 2020.

9. Sichuan launched a level-1 public health emergency response. http://www.sc. xinhuanet.com/content/2020-01/25/c_1125500856.htm. Accessed 9 Mar 2020.

10. Notice of General Office of the People's Government of Sichuan Province on Issuing Level I Emergency Measures against Pneumonia Associated with the Novel Coronavirus. http://www.sc.gov.cn/zcwj/xxgk/NewT.aspx?i=202 00125200454-361030-00-000. Accessed 17 Mar 2020.

11. Emergency Notice of 3 Departments Including Administration for Market Regulation of Sichuan Province on Strengthening the Supervision of Massive Dinner Gatherings during the COVID-19 Prevention and Control
Period. http://scjgj.sc.gov.cn/scjgj/c104492/2020/1/25/9c4ce2cc023d460384 bbc0b6697339a0.shtml. Accessed 17 Mar 2020.

12. Announcement of Sichuan Headquarters for Emergency Response to the COVID-19 Epidemic. http://www.sc.gov.cn/zcwj/xxgk/NewT.aspx?i=2020012 8192811-452803-00-000. Accessed 17 Mar 2020.

13. Sichuan Headquarters for Emergency Response to the COVID-19 Epidemic. Notice on Issuing Eight Measures for Strengthening Massive Dinner Gatherings in Rural Areas during the COVID-19 Prevention and Control Period. http://www.sc.gov.cn/Zcwj/xxgk/NewT.aspx?i=202002082 01515-635233-00-000. Accessed 17 Mar 2020.

14. Bi QF, Wu YS, Mei SJ, Ye CF, Zou X, Zhang Z, et al. Epidemiology and Transmission of COVID-19 in Shenzhen China: Analysis of 391 cases and 1,286 of their close contacts. medRxiv. 2020; 2020.2003.2003.20028423. https://doi.org/https://doi.org/10.1101/2020.03.03.20028423.

15. Liu ZY, Gao LD, Hu SX, Luo KW, Xiao JH, Zhao SL, et al. Diagnosis and treatment of 697 confirmed cases of coronavirus disease 2019 in Hunan province [in Chinese]. Pract Prev Med. 2020;27 http://kns.cnki.net/kcms/ detail/43.1223.R.20200305.1921.008.html.

16. Li Q, Guan XH, Wu P, Wang XY, Zhou L, Tong YQ, et al. Early transmission dynamics in Wuhan, China, of novel coronavirus-infected pneumonia. New Engl J Med 2020. https://doi.org/https://doi.org/10.1056/nejmoa2001316.

17. Backer JA, Klinkenberg D, Wallinga J. Incubation period of 2019 novel coronavirus (2019-nCoV) infections among travellers from Wuhan, China, 20-28 January 2020. Euro Surveill. 2020;25(5). https://doi.org/https://doi.org/ 10.2807/1560-7917.es.2020.25.5.2000062.

18. Guan WJ, Ni ZY, Hu Y, Liang WH, Ou CQ, He JX, et al. Clinical characteristics of coronavirus disease 2019 in China. New Engl J Med 2020. https://doi.org/ https://doi.org/10.1056/nejmoa2002032.

19. Qian GQ, Yang NB, Ding F, Ma AHY, Wang ZY, Shen YF, et al. Epidemiologic and clinical characteristics of 91 hospitalized patients with COVID-19 in Zhejiang, China: a retrospective, multi-Centre case series. QJM 2020. https:// doi.org/https://doi.org/10.1093/qjmed/hcaa089.

20. Wang P, Lu JA, Jin YY, Zhu MF, Wang LL, Chen S.J. Epidemiological characteristics of 1212 COVID-19 patients in Henan, China. medRxiv. 2020; 2020.02.21.20026112. https://doi.org/https://doi.org/10.1101/2020.02.21. 20026112.

21. Bai $Y$, Yao L, Wei T, Tian F, Jin DY, Chen $L$, et al. Presumed asymptomatic carrier transmission of COVID-19. JAMA. 2020. https://doi.org/https://doi.org/ 10.1001/jama.2020.2565

22. Tong ZD, Tang A, Li KF, Li P, Wang HL, Yi JP, et al. Potential Presymptomatic Transmission of SARS-CoV-2, Zhejiang Province, China, 2020. Emerg Infect Dis. 2020;26(5). https://doi.org/https://doi.org/10.3201/eid2605.200198.

23. Gao WJ, Li LM. Advances on presymptomatic asymptomatic carrier transmission of COVID-19 [in Chinese]. Chin J Epidemiol2020;41(4): 485-488. https://doi.org/https://doi.org/10.3760/cma.j.cn112338-20200228-00207.

24. Huang CL, Wang YM, Li XW, Ren L, Zhao JP, Hu Y, et al. Clinical features of patients infected with 2019 novel coronavirus in Wuhan, China Lancet 2020; 395(10223):497-506. https://doi.org/https://doi.org/10.1016/s01406736(20)30183-5.

25. Chen NS, Zhou M, Dong X, Qu JM, Gong FY, Han Y, et al. Epidemiological and clinical characteristics of 99 cases of 2019 novel coronavirus pneumonia in Wuhan, China: a descriptive study. Lancet. 2020;395(10223):507-513. https://doi.org/https://doi.org/10.1016/s0140-6736(20)30211-7.

26. Novel Coronavirus Pneumonia Emergency Response Epidemiology Team. The epidemiological characteristics of an outbreak of 2019 novel coronavirus disease (COVID-19) in China [in Chinese]. Chin J Epidemiol 2020; 41(2):145-151. https://doi.org/https://doi.org/10.3760/cma.j.issn.0254-6450. 2020.02.003.

27. Horton R. Offline: 2019-nCoV outbreak-early lessons. Lancet. 2020;395(10221): 322. https://doi.org/https://doi.org/10.1016/s0140-6736(20)30212-9.

28. Huang WL. Molecular Virology. 3th ed. Chaoyang District: People's Medical Publishing House; 2016.

29. Jia WX. Medical Microbiology. 2th ed. Chaoyang District: People's Medical Publishing House; 2010.

30. Long QX, Tang XJ, Shi QL, Li Q, Deng HJ, Yuan J, et al: Clinical and immunological assessment of asymptomatic SARS-CoV-2 infections. Nat Med 2020. https://doi.org/https://doi.org/10.1038/s41591-020-0965-6.

\section{Publisher's Note}

Springer Nature remains neutral with regard to jurisdictional claims in published maps and institutional affiliations. 